\title{
Stator Fault Detection in Induction Motors by Autoregressive Modeling
}

\author{
Francisco M. Garcia-Guevara, ${ }^{1}$ Francisco J. Villalobos-Piña, ${ }^{1}$ Ricardo Alvarez-Salas, ${ }^{2}$ \\ Eduardo Cabal-Yepez, ${ }^{3}$ and Mario A. Gonzalez-Garcia ${ }^{2}$ \\ ${ }^{1}$ Departamento de Ingeniería Electronica, Instituto Tecnologico de Aguascalientes, Avenida Adolfo Lopez Mateos No. 1801 Oriente, \\ Aguascalientes, AGS, Mexico \\ ${ }^{2}$ CIEP, Facultad de Ingeniería, Universidad Autónoma de San Luis Potosí, Avenida Dr. Manuel Nava No. 8, San Luis Potosí, \\ SLP, Mexico \\ ${ }^{3}$ Departamento de Estudios Multidisciplinarios, Division de Ingenierías Campus Irapuato-Salamanca (DICIS), \\ Universidad de Guanajuato, 38944 Yuriria, GTO, Mexico
}

Correspondence should be addressed to Ricardo Alvarez-Salas; ralvarez@uaslp.mx

Received 24 November 2015; Revised 2 March 2016; Accepted 8 March 2016

Academic Editor: Peter Dabnichki

Copyright ( 2016 Francisco M. Garcia-Guevara et al. This is an open access article distributed under the Creative Commons Attribution License, which permits unrestricted use, distribution, and reproduction in any medium, provided the original work is properly cited.

\begin{abstract}
This study introduces a novel methodology for early detection of stator short circuit faults in induction motors by using autoregressive (AR) model. The proposed algorithm is based on instantaneous space phasor (ISP) module of stator currents, which are mapped to $\alpha$ - $\beta$ stator-fixed reference frame; then, the module is obtained, and the coefficients of the AR model for such module are estimated and evaluated by order selection criterion, which is used as fault signature. For comparative purposes, a spectral analysis of the ISP module by Discrete Fourier Transform (DFT) is performed; a comparison of both methodologies is obtained. To demonstrate the suitability of the proposed methodology for detecting and quantifying incipient short circuit stator faults, an induction motor was altered to induce different-degree fault scenarios during experimentation.
\end{abstract}

\section{Introduction}

The fault diagnosis for electrical machines has become far more critical as the population of electric machines has greatly increased in recent years. The total number of operating electrical machines in the world was around 16.1 billion in 2011 , with a growth rate of about $50 \%$ in the last five years [1].

Three-phase induction motors represent the principal source of movement in the electrical and processing industry [2]. This type of motors has an exclusive position in the energy conversion and they are responsible of almost $90 \%$ of the electric energy consumed by electric machines. Three-phase induction motors correspond to nearly $60 \%$ of all electric machines [3].

The success of three-phase induction is due to the low cost, robustness, and high performance in variable speed tasks; this has been achieved through the development of new control laws and more versatile semiconductor devices [4]. However, most control algorithms may not be reliable when a fault condition occurs $[1,2]$.

Stator short circuit fault diagnosis in three-phase induction motors represents a significant percentage of the electrical machine defects [5]. In this category there are faults in the stator-winding such as short circuits among turns and the magnetic circuit. In the first case, the internal asymmetry will cause the circulation of high currents in the portion of the stator-winding affected by the fault, this contributes to the degradation of other portions of the winding, and the remaining time between the onset of fault and the failure depends on various factors such as the initial number of shorted turns, winding configuration, the power and voltage 
TABLE 1: Comparison of IEEE, EPRI, and Allianz surveys.

\begin{tabular}{lccc}
\hline \multirow{2}{*}{ Component } & \multicolumn{3}{c}{ Fault percent distribution } \\
& IEEE & EPRI & Allianz \\
\hline Bearings & 44 & 41 & 13 \\
Stator & 26 & 36 & 63 \\
Rotor & 8 & 9 & 13 \\
Others & 22 & 14 & 8 \\
\hline
\end{tabular}

achieved, and environmental conditions among others [6]. Therefore early detection is critical.

Unfortunately the induction motor may fail due to other mechanisms, such as bearings [7], the rotor due cracks in short-circuiting rings or broken bars [8], and eccentricity in the air gap [9]. In [10, 11], some of the techniques commonly used for this purpose are shown.

A comparative fault distribution informed by [12] is shown in Table 1; this comparison was carried out in several surveys of faults of large induction motors, conducted by the Institute of Electrical and Electronics Engineers (IEEE) [13-15], Electric Power Research Institute (EPRI) [16], and Allianz [17]. Differences in distributions are because survey conducted by IEEE and EPRI focuses on medium-sized induction machines, while Allianz survey focuses more on medium- to high-voltage large induction machines.

Spectral analysis has been widely used for diagnosis of electric and mechanical faults $[18,19]$. Autoregressive (AR) models characterize spectral and energy properties of a system by representing them as time series, which consider each sample in the time domain as a linear combination of previous sampled values in the presence of white noise with a Gaussian distribution [20]. Therefore, the coefficients of a univariate AR model are the autocorrelations of the time series, which represent a dependence measure of the current value with respect to previous values, and provide information of the system dynamics in time. When a fault occurs in a system, its dynamics changes; therefore, the AR model coefficients change too. This suggests that a parameter obtained from these coefficients can be used as a fault diagnosis tool for induction machines.

The use of AR model coefficients has been reported in some works; for instance, in [21, 22] bearing faults are detected, and in $[23,24]$ faults in gearbox coupling systems are detected. In these works, coefficients are used to estimate the power spectral density (PSD) from the transfer function of the AR model. The harmonic analysis allows detecting those faults that cause atypical frequencies and parasitic oscillations [25].

This paper presents a novel stator fault detection technique based on AR model of the ISP module for the stator $\alpha-\beta$ reference frame from the Concordia transformation, which is evaluated via the Akaike Final Prediction Error (FPE) criterion to obtain an indicator of the induction motor operational condition.

\section{Fault Detection Scheme}

2.1. AR Model. The proposed electric stator fault diagnosis scheme consists in acquiring $N$ samples of the induction motor three phase stator currents $i_{a}, i_{b}$, and $i_{c}$ :

$$
\left[\begin{array}{l}
i_{a} \\
i_{b} \\
i_{c}
\end{array}\right]=\left[\begin{array}{llll}
i_{a 1} & i_{a 2} & \cdots & i_{a N} \\
i_{b 1} & i_{b 2} & \cdots & i_{b N} \\
i_{c 1} & i_{c 2} & \cdots & i_{c N}
\end{array}\right] .
$$

The three currents are mapped into the $\alpha$ - $\beta$ stator-fixed reference frame using Concordia transformation:

$$
\left[\begin{array}{l}
i_{\alpha} \\
i_{\beta} \\
i_{o}
\end{array}\right]=\frac{1}{\sqrt{2}}\left[\begin{array}{ccc}
1 & \cos \left(-\frac{2 \pi}{3}\right) & \cos \left(\frac{2 \pi}{3}\right) \\
0 & -\sin \left(-\frac{2 \pi}{3}\right) & -\sin \left(\frac{2 \pi}{3}\right) \\
\frac{1}{\sqrt{2}} & \frac{1}{\sqrt{2}} & \frac{1}{\sqrt{2}}
\end{array}\right]\left[\begin{array}{l}
i_{a} \\
i_{b} \\
i_{c}
\end{array}\right] .
$$

Then, ISP module [26] is obtained for each sample of $i_{\alpha}$ and $i_{\beta}$ by

$$
\text { ISP }=\left\|i_{\alpha \beta}\right\|=\sqrt{i_{\alpha}^{2}+i_{\beta}^{2}} .
$$

From the ISP module the coefficients of a $p$-order AR model are estimated. The AR model is a finite impulse response filter (FIR) that can be defined as follows:

$$
\mathrm{ISP}_{n}=\sum_{k=1}^{p} A_{k} \operatorname{ISP}_{n-k}+\varepsilon_{n}, \quad n=1, \ldots, N,
$$

where $A_{k}$ contains the coefficients of the AR model and ISP $_{n-k}$ is the value of the ISP module delayed $k$ samples. Finally, $\varepsilon_{n}$ represents uncorrelated random noise.

AR model coefficients can be estimated by diverse methods like the solution of Yule-Walker equations [27], the Burg method [28], or the covariance method [29]. In this work the coefficients are calculated by least squares approximation algorithm [30], where the p-order AR model (4) is estimated in the form of a regression model expressed as

$$
\operatorname{ISP}_{n}=A_{p} u_{n}+\varepsilon_{n} \quad \text { with } \varepsilon_{n}=\operatorname{noise}(C)
$$

with a coefficients vector

$$
A_{1}, \ldots, A_{p}
$$

and with predictors

$$
u_{n}=\left[\begin{array}{llll}
1 & \operatorname{ISP}_{n-1} & \cdots & \operatorname{ISP}_{n-p}
\end{array}\right]^{T} .
$$

In terms of moments

$$
\begin{gathered}
U=\sum_{n=1}^{N} u_{n} u_{n}^{T}, \\
\mathrm{ISP}=\sum_{n=1}^{N} \operatorname{ISP}_{n} \operatorname{ISP}_{n}^{T}, \\
W=\sum_{n=1}^{N} \operatorname{ISP}_{n} u_{n}^{T} .
\end{gathered}
$$


The least squared estimates of coefficients $\widehat{A}$ are estimated by

$$
\widehat{A}=W U^{-1} \text {. }
$$

The residual variance

$$
\widehat{C}=\frac{1}{N-p} \sum_{n=1}^{N} \widehat{\varepsilon}_{n} \widehat{\varepsilon}_{n}^{T} \text { with } \widehat{\varepsilon}_{n}=\operatorname{ISP}_{n}-\widehat{A}_{p} u_{n}
$$

is an estimate of the noise covariance $C$ and can be expressed as

$$
\widehat{C}=\frac{1}{N-p+1}\left(\text { ISP }-W U^{-1} W^{T}\right) .
$$

The residual variance is proportional to Schur complement of the matrix

$$
\Gamma=\left[\begin{array}{cc}
U & W^{T} \\
W & \text { ISP }
\end{array}\right]=\sum_{n=1}^{N}\left[\begin{array}{c}
u_{n} \\
\operatorname{ISP}_{n}
\end{array}\right]\left[\begin{array}{ll}
u_{n}^{T} & \operatorname{ISP}_{n}^{T}
\end{array}\right]
$$

which is the moment matrix $\Gamma=K^{T} K$ belonging to the data matrix

$$
K=\left[\begin{array}{cc}
u_{1}^{T} & \mathrm{ISP}_{1}^{T} \\
\vdots & \vdots \\
u_{N}^{T} & \mathrm{ISP}_{N}^{T}
\end{array}\right],
$$

where the data matrix $K \in \mathfrak{R}^{N-p \times p+1}$.

The least squares estimation (9) is obtained from a QR factorization of $K$ through the Householder algorithm:

$$
K=Q R,
$$

where $Q$ and $R$ are orthogonal upper triangular matrices, and $R$ is given by

$$
R=\left[\begin{array}{cc}
R_{11} & R_{12} \\
0 & R_{22}
\end{array}\right]
$$

where $R \in \mathscr{R}^{N+1 \times p}, R_{11} \in \mathscr{R}^{p \times p}, R_{12} \in \mathscr{R}^{1 \times p}$, and $R_{22} \in \mathscr{R}$.

The QR factorization of the data matrix (14) leads to the Cholesky factorization

$$
\Gamma=K^{T} K=R^{T} R
$$

of the moment matrix [31]

$$
\left[\begin{array}{cc}
U & W^{T} \\
W & \text { ISP }
\end{array}\right]=R^{T} R=\left[\begin{array}{cc}
R_{11}^{T} R_{11} & R_{11}^{T} R_{12} \\
R_{12}^{T} R_{11} & R_{12}^{T} R_{12}+R_{22}^{2}
\end{array}\right] .
$$

The least squares estimation of the residual variance $\widehat{C}$ and the parameter vector $\widehat{A}$ are computed from the Cholesky factorization by

$$
\begin{aligned}
\widehat{C} & =\frac{R_{22}^{2}}{N-p+1} . \\
\widehat{A} & =R_{11}^{-1} R_{12}^{T} .
\end{aligned}
$$

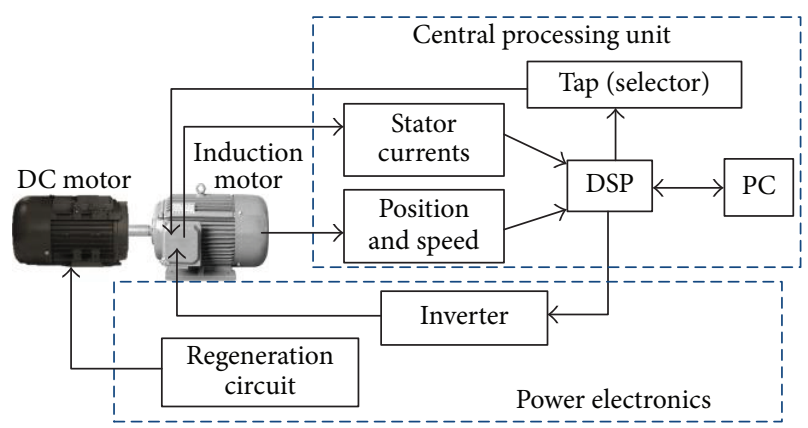

FIGURE 1: Block diagram of experimental setup.

Regardless of the used method, it is necessary to define the model order that best fits the signal of interest (3). The chosen order selection criterion was the FPE which was evaluated for a $p$-order AR model by

$$
\mathrm{FPE}=\log \left(R_{22}^{2}\right)-\log \left(\frac{N(N-p+1)}{N+p+1}\right) .
$$

Finally, the fluctuations in FPE values for different cases with and without fault are compared.

2.2. Frequency Scheme. The proposed method was compared with a frequency domain analysis using the Discrete Fourier Transform (DFT) algorithm. The stator fault spectral signature $f_{\text {est }}$ of the ISP module occurs in the integer multiples of twice the voltage frequency applied to the motor $f_{l}$ :

$$
f_{\text {est }}=2 m f_{l},
$$

where $m=1,2, \ldots, \infty$ [32]. Since components of the fault spectral signature decrease while $m$ increases, just the main component $(m=1)$ is considered; hence

$$
f_{\text {est }}=2 f_{l} \text {. }
$$

\section{Experimental Setup}

The proposed methodology was validated on an experimental platform. The implemented test bed for performing the experimental analysis is depicted in Figure 1. It consists in a $3 \mathrm{HP}$, three-phase induction motor altered to introduce different electric stator fault scenarios; a regeneration system based on a direct current (DC) motor was used for adding load torque; a full bridge three-phase inverter was used for varying the motor rotating speed; a Hall-effect based data acquisition system was used for acquiring the stator currents; the induction motor was instrumented with an optical encoder to acquire the rotor angular position and speed shaft; and a central processing unit (CPU) based on TI TMS320F28335 32-bit floating point digital signal processor (DSP) was used for performing the necessary control and measurement functions.

The CPU received a group of commands from a personal computer (PC) to acquire and control the instrumentation of a power electronic system on the test bed. 


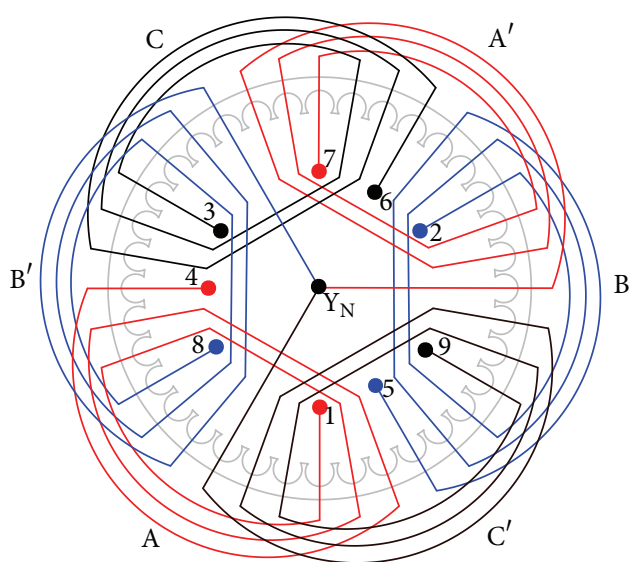

FIGURE 2: Three-phase induction motor stator-winding structure.

The stator currents were acquired and stored in the memory of an IRAM DSP, taking 4096-sample frames from each phase. The acquired signals were sent to a $\mathrm{PC}$ via high speed USB. A computer-program based on Delphi language was used to perform the fault diagnosis analysis.

In order to insert different types of stator faults, like interturn short circuit or open circuit in one phase, the induction machine was rewinded. The schematic diagram of the induction motor is shown in Figure 2.

The electric structure for the stator-winding comprises 6 groups of coils, 2 groups per phase (A, $\mathrm{A}^{\prime}, \mathrm{B}, \mathrm{B}^{\prime}$, and $\mathrm{C}, \mathrm{C}^{\prime}$ ) distributed in 36 slots, and three concentric coils per group of 45 turns each, with three 22-AGW wires for a total of 7290 turns.

The low-voltage operational condition for the induction motor was achieved by connecting in parallel the double star windings and supplying the phase voltages $V_{\mathrm{AN}}$ to terminals 1 and $7, V_{\mathrm{BN}}$ to 2 and 8 , and $V_{\mathrm{CN}}$ to 3 and 9 and joining terminals 4,5 , and 6 to conform the neutral star $Y_{N}$, as specified by the manufacturer.

The fault insertion in the stator-winding is accomplished by connecting or disconnecting some segments of phase $\mathrm{C}$ winding to phase voltage $V_{\mathrm{CN}}$ or neutral $\mathrm{Y}_{\mathrm{N}}$, using tap derivations $\mathrm{C}_{1}, \ldots, \mathrm{C}_{8}$. This is achieved by an insulated electric circuit containing a group of relays that are managed by the DSP-based control system to select the fault severity in the motor. Possible connections are depicted with dotted lines in Figure 3.

The characterization of the induction motor parameters was performed at line frequency $f_{l}(60 \mathrm{~Hz})$ via the threevoltmeter method, which consists in inserting a pattern resistance $R_{P}$ in each phase of the motor and measuring stator voltages $V_{1}, V_{2}$, and $V_{3}$ and currents as shown in Figure 4; then, the impedance $Z_{L}$, inductive reactance $X_{L}$, resistance $R_{L}$, and inductance $L$ for each phase are calculated. The results are shown in Table 2.

\section{Experimental Results}

A comparative analysis was performed between the FPE of the AR model coefficients and fault spectral signature for

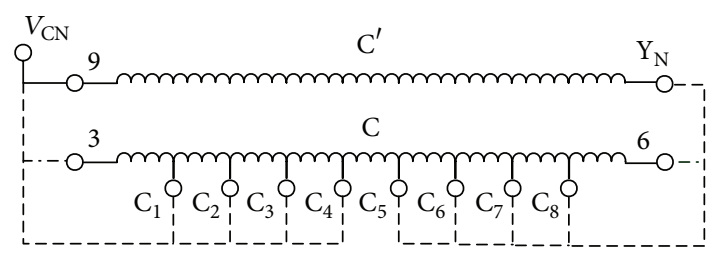

FIGURE 3: Modified stator-winding with tap derivations insertion.

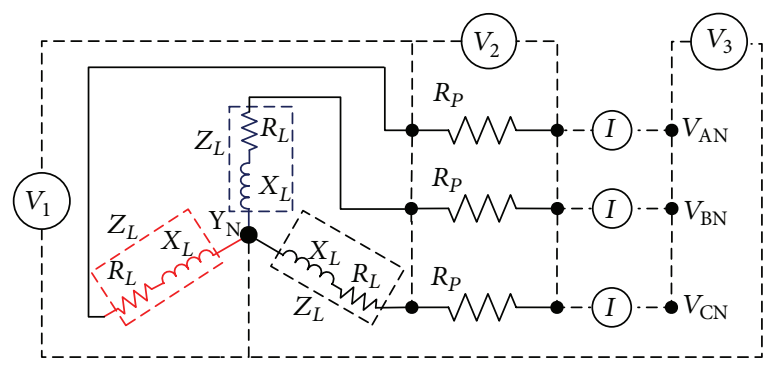

FIGURE 4: Characterization of the induction stator-winding parameters.

TABLE 2: Stator-winding parameters.

\begin{tabular}{lccc}
\hline Parameter & Phase A & Phase B & Phase C \\
\hline$L(\mathrm{mH})$ & 5.055 & 5.025 & 5.035 \\
$Z_{L}(\Omega)$ & 1.909 & 1.899 & 1.902 \\
$X_{L}(\Omega)$ & 1.905 & 1.894 & 1.898 \\
$R_{L}(\Omega)$ & 0.127 & 0.128 & 0.126 \\
\hline
\end{tabular}

TABLE 3: Stator-winding parameters for healthy and fault cases.

\begin{tabular}{lccc}
\hline $\begin{array}{l}\text { Phase C } \\
\text { parameter }\end{array}$ & Healthy & $\begin{array}{c}\text { Case 1 } \\
\Delta \%\end{array}$ & $\begin{array}{c}\text { Case 2 } \\
\Delta \%\end{array}$ \\
\hline$L(\mathrm{mH})$ & 5.035 & 6.494 & 8.639 \\
$Z_{L}(\Omega)$ & 1.902 & 6.519 & 8.622 \\
$X_{L}(\Omega)$ & 1.898 & 6.480 & 8.640 \\
$R_{L}(\Omega)$ & 0.126 & 7.936 & 12.698 \\
\hline
\end{tabular}

ISP module using DFT. Twenty trials were performed on each one of the treated conditions. Three different states were considered: healthy case and two distinct-degree stator fault conditions as shown in Table 3. The healthy condition is obtained by $V_{\mathrm{CN}}$ to terminal 3 and $\mathrm{Y}_{\mathrm{N}}$ to terminal 6 (see Figure 3 ). In the first faulty condition (three turns), $V_{\mathrm{CN}}$ is connected to $\mathrm{C}_{1}$ terminal. In the second faulty condition (nine turns) $\mathrm{Y}_{\mathrm{N}}$ is connected to $\mathrm{C}_{8}$ terminal. Both faulty cases correspond to incipient short circuit stator faults.

The defect level for each faulty condition is indicated through a change in the percentage ratio $(\Delta \%)$ of their corresponding coefficient magnitudes with respect to the healthy case.

Experimental data were obtained from the ISP module. For each acquired stator current, 4096 samples were obtained at the sampling frequency $f_{s}=4000 \mathrm{~Hz}$. A load torque of $0.4 \mathrm{Nm}$ was applied for all experiments at different speeds: 


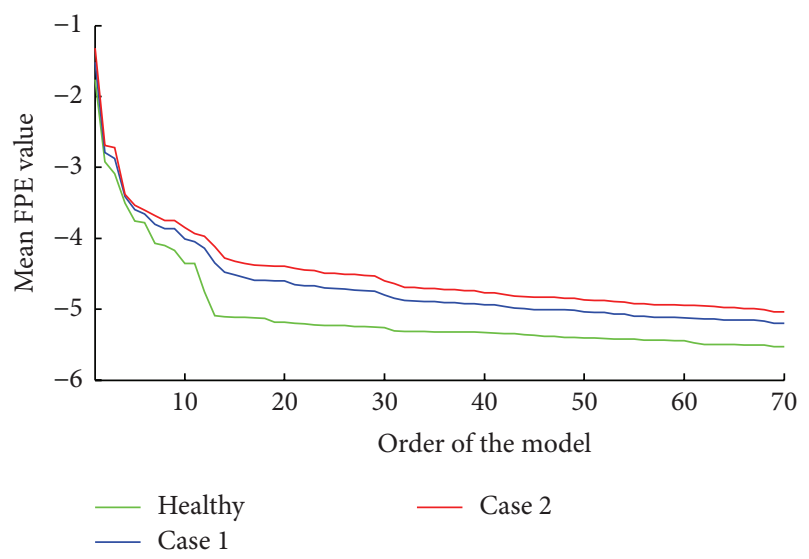

FIGURE 5: Mean FPE value for three fault scenarios at a speed of $1800 \mathrm{rpm}$.

TABLE 4: Mean FPE value of order $p=35$ for three fault scenarios at different speeds.

\begin{tabular}{lccc}
\hline$f_{l}(\mathrm{~Hz})$ & Healthy & Case 1 & Case 2 \\
& & $\Delta \%$ & $\Delta \%$ \\
\hline 60 & -5.1151 & 8.06 & 11.59 \\
50 & -5.2309 & 5.70 & 7.63 \\
40 & -5.3140 & 5.51 & 7.26 \\
\hline
\end{tabular}

$1200 \mathrm{rpm}\left(f_{l}=40 \mathrm{~Hz}\right), 1500 \mathrm{rpm}\left(f_{l}=50 \mathrm{~Hz}\right)$, and $1800 \mathrm{rpm}$ $\left(f_{l}=60 \mathrm{~Hz}\right)$.

4.1. AR Model Approach. The mean FPE values of the ISP module from the three treated cases at rotating speed of $1800 \mathrm{rpm}\left(f_{l}=60 \mathrm{~Hz}\right)$, evaluated for different orders $(p=$ $2, \ldots, 70)$, are shown in Figure 5 . It can be observed that the mean FPE value decreases for the three cases when the AR model order increases. The gap among the values for orders $(p=3, \ldots, 8)$ is minimum; however, the gap increases from order $p=11$ and it remains as the order goes up.

The mean of FPE value and their percentage rate of change $\Delta \%$ among different fault degrees with respect to the healthy case at three different speeds for a 35-order AR model are summarized in Table 4.

4.2. Spectra Analysis. Figure 6 shows the stator fault spectral signature corresponding to the three considered conditions reported in Table 5 , at the rotating speed of $1800 \mathrm{rpm}\left(f_{l}=\right.$ $60 \mathrm{~Hz}$ ). From this figure, it can be observed that the frequency component at $120 \mathrm{~Hz}$ increases its magnitude with respect to the fault level. Table 5 summarizes $\Delta \%$ average percentage rate of the fault magnitude of characteristic frequency components for the different fault scenarios in comparison with the healthy case at three different speeds.

4.3. Discussion of Results. From the obtained results, the proposed methodology does not require a power-two $N$ or
TABLE 5: Mean value of the spectrum signature for healthy and faulty cases at different rotating speeds.

\begin{tabular}{lccc}
\hline$f_{l}(\mathrm{~Hz})$ & Healthy & $\begin{array}{c}\text { Case } 1 \\
\Delta \%\end{array}$ & $\begin{array}{c}\text { Case 2 } \\
\Delta \%\end{array}$ \\
\hline 60 & 0.045 & 583.59 & 858.21 \\
50 & 0.033 & 568.77 & 856.36 \\
40 & 0.047 & 511.59 & 756.01 \\
\hline
\end{tabular}

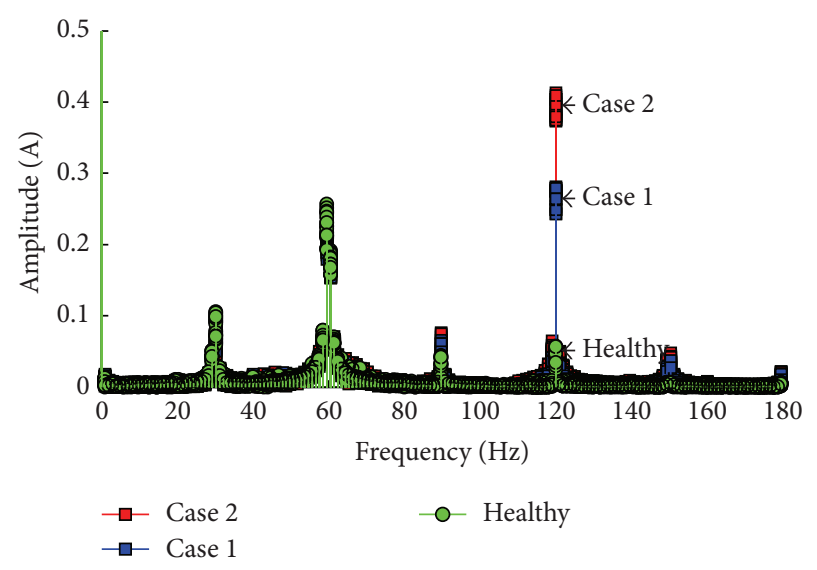

FIGURE 6: Spectrum for healthy and faulty cases at $1800 \mathrm{rpm}$.

adjusting the sampling frequency $f_{s}$ depending on the motor rotating speed. On the other hand, the common spectral analysis technique for detecting fault signatures does require $f_{s}$ to be a multiple of $f_{l}$ [28]; furthermore, in the case of $N$ not being a power of two, zero padding must have to be done, and the technique will fail.

For a fixed $p$-order AR model with a defined sampling period, for instance, $p=35$ and $N=4096$, the FPE computation and estimation of the AR model coefficients will require $(N+p+2)(p)^{2}=5,061,700$ operations [30] for solving the linear equation system with real numbers. The proposed approach was executed on an Intel $2.5 \mathrm{GHz}$ processor i7$4710 \mathrm{HQ}$, and it took around 0.00401 seconds, whereas the DFT technique requires $N^{2}=16,777,216$ operations and takes 0.0129 seconds, under exactly the same conditions, involving the product of exponential functions with complex numbers.

The downward trend in the mean FPE value as the model order increases indicates that the higher the order of the AR model, the more accurate the fault estimation; however, the computational burden will also increase. Nevertheless, for a model order $p \geq 12$ (see Figure 5) a tendency to keep each operational condition separated is clear. Hence, for diagnosis purposes, this indicates that it is not necessary to select a very high-order AR model.

On the other hand, it was possible to detect the presence of fault conditions even with low order models $(p=1)$, but it was not possible to quantify the magnitude of the fault, whereas, for a higher-order model and given the scope of this research that only characterized the electrical stator 


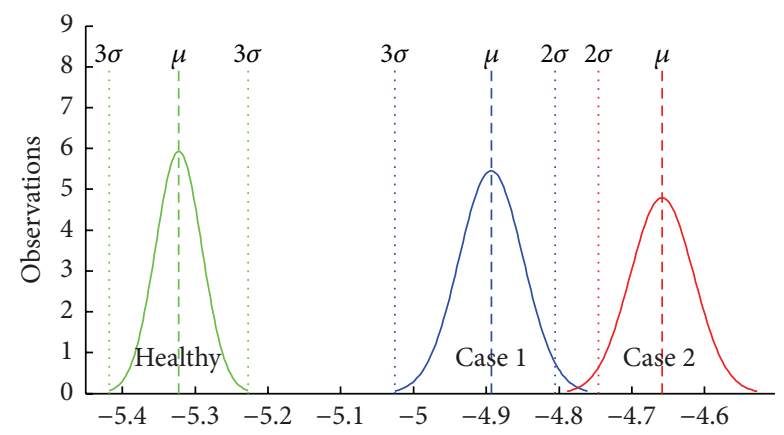

FIGURE 7: Experimental FPE normal distributions.

parameters for $f_{l}=60 \mathrm{~Hz}$, it was shown that there is approximated proportionality between the fault level and the inductance value.

For instance, in the first faulty case the increment for the three the FPE value $8.06 \%$ with respect to the healthy condition the inductance value and the $7.6 \%$ obtained by three voltmeter method. While for the second case, it was of $11.5 \%$ against $8.6 \%$, so that in a future work it will be necessary to characterize the induction motor at different frequencies and in order to verify whether it is possible to quantify the level of damage, without evaluating the transfer function of the AR model in the frequency domain as it is commonly used in literature.

To demonstrate the effectiveness of the proposed methodology, a 3-sigma rule was applied on 20 experiments for each of the different treated conditions. The obtained results are depicted in Figure 7; from this figure and due to the fact that the Gaussian distributions do not overlap in their corresponding 3-sigma rule interval for (healthy case and case 1), a confidence level of more than $99.7 \%$ is endured on the detection and $95 \%$ in the identification of stator faults.

\section{Conclusions}

Electric motors are responsible for around $90 \%$ of the electric-machine energy consumption. Stator faults are very important because of the effects on the performance of the induction machine; hence, several studies are available for induction motor fault detection. In this regard, this work proposed a novel methodology based on the use of FPE for stator fault detection in induction motors. The introduced approach was experimentally validated considering three different operational conditions: healthy case and two different-degree stator faults. From the obtained results the usefulness and efficiency of the proposed method for fault detection and quantification of incipient short circuit stator faults, considering low AR model orders for different faulty conditions, were demonstrated considering a 3 -sigma rule.

\section{Competing Interests}

The authors declare that they have no competing interests.

\section{Acknowledgments}

This work was partially supported by the Doctorado en Ciencias de la Ingeniería of the Instituto Tecnologico de Aguascalientes and the PROMEP under Grants PROMEP/ITA/ 103.5/13/8890 and PROMEP/UASLP/CARepPrel-15 and Universidad Autónoma de San Luis Potosí under Grant C14-FAI04-42.42. Mario A. Gonzalez-Garcia is a Research Fellow of the National Council of Science and Technology (CONACyT), Mexico.

\section{References}

[1] H. A. Toliyat, S. Nandi, S. Choi, and H. Meshging-Kelk, Electric Machines, CRC Press, Boca Raton, Fla, USA, 2013.

[2] H. Henao, G.-A. Capolino, M. Fernandez-Cabanas et al., "Trends in fault diagnosis for electrical machines: a review of diagnostic techniques," IEEE Industrial Electronics Magazine, vol. 8, no. 2, pp. 31-42, 2014.

[3] S. Nandi, H. A. Toliyat, and X. Li, "Condition monitoring and fault diagnosis of electrical motors-a review," IEEE Transactions on Energy Conversion, vol. 20, no. 4, pp. 719-729, 2005.

[4] F. Betin, G.-A. Capolino, D. Casadei et al., "Trends in electrical machines control: samples for classical, sensorless, and faulttolerant techniques," IEEE Industrial Electronics Magazine, vol. 8, no. 2, pp. 43-55, 2014.

[5] C. H. De Angelo, G. R. Bossio, S. J. Giaccone, M. I. Valla, J. A. Solsona, and G. O. García, "Online model-based statorfault detection and identification in induction motors," IEEE Transactions on Industrial Electronics, vol. 56, no. 11, pp. 46714680, 2009.

[6] S. M. A. Cruz and A. J. M. Cardoso, "Multiple reference frames theory: a new method for the diagnosis of stator faults in three-phase induction motors," IEEE Transactions on Energy Conversion, vol. 20, no. 3, pp. 611-619, 2005.

[7] Z. Yang, "Automatic condition monitoring of industrial rollingelement bearings using motor's vibration and current analysis," Shock and Vibration, vol. 2015, Article ID 486159, 12 pages, 2015.

[8] H.-Y. Zhu, J.-T. Hu, L. Gao, and H. Huang, "Practical aspects of broken rotor bars detection in PWM voltage-source-inverterfed squirrel-cage induction motors," Journal of Applied Mathematics, vol. 2013, Article ID 128368, 11 pages, 2013.

[9] R. Shnibha, A. Albarbar, A. Abouhnik, and G. Ibrahim, "A more reliable method for monitoring the condition of three-phase induction motors based on their vibrations," ISRN Mechanical Engineering, vol. 2012, Article ID 230314, 9 pages, 2012.

[10] A. H. Bonnett and C. Yung, "Increased efficiency versus increased reliability," IEEE Industry Applications Magazine, vol. 14, no. 1, pp. 29-36, 2008.

[11] M. Hernandez-Vargas, E. Cabal-Yepez, and A. Garcia-Perez, "Real-time SVD-based detection of multiple combined faults in induction motors," Computers \& Electrical Engineering, vol. 40, no. 7, pp. 2193-2203, 2014.

[12] P. Zhang, Y. Du, T. G. Habetler, and B. Lu, "A survey of condition monitoring and protection methods for medium-voltage induction motors," IEEE Transactions on Industry Applications, vol. 47, no. 1, pp. 34-46, 2011.

[13] R. N. Bell, D. W. McWilliams, P. O’Donnell, C. Singh, and S. J. Wells, "Report of large motor reliability survey of industrial and commercial installations, part I," IEEE Transactions on Industry Applications, vol. IA-21, no. 4, pp. 853-864, 1985. 
[14] "Report of large motor reliability survey of industrial and commercial installations, Part II," IEEE Transactions on Industry Applications, vol. 21, no. 4, pp. 865-872, 1985.

[15] "Report of large motor reliability survey of industrial and commercial installations: part 3," IEEE Transactions on Industry Applications, vol. 21, no. 4, pp. 865-872, 1987.

[16] P. F. Albrecht, J. C. Appiarius, R. M. McCoy, E. L. Owen, and D. K. Sharma, "Assessment of the reliability of motors in utility applications-updated," IEEE Transactions on Energy Conversion, vol. 1, no. 1, pp. 39-46, 1986.

[17] H. O. Seinsch, "Monitoring und diagnose elektrischer maschinen und antriebe," in Proceedings VDE Workshop, Allianz Schadensstatistik an HS Motoren, 1996-1999, VDE, 2001.

[18] L.-L. Jiang, H.-K. Yin, X.-J. Li, and S.-W. Tang, "Fault diagnosis of rotating machinery based on multisensor information fusion using SVM and time-domain features," Shock and Vibration, vol. 2014, Article ID 418178, 8 pages, 2014.

[19] P. A. Delgado-Arredondo, A. Garcia-Perez, D. Morinigo-Sotelo et al., "Comparative study of time-frequency decomposition techniques for fault detection in induction motors using vibration analysis during startup transient," Shock and Vibration, vol. 2015, Article ID 708034, 14 pages, 2015.

[20] J. Morton, P. A. Torrione, and L. M. Collins, "Variational Bayesian learning for mixture autoregressive models with uncertain-order," IEEE Transactions on Signal Processing, vol. 59, no. 6, pp. 2614-2627, 2011.

[21] G. Wang, Z. Luo, X. Qin, Y. Leng, and T. Wang, "Fault identification and classification of rolling element bearing based on time-varying autoregressive spectrum," Mechanical Systems and Signal Processing, vol. 22, no. 4, pp. 934-947, 2008.

[22] C. Liu, X. Zhou, S. Yang, W. Liang, and Q. Miao, "Cooling fan bearing diagnosis based on AR\& MED," in Proceedings of the International Conference on Quality, Reliability, Risk, Maintenance, and Safety Engineering (ICQR2MSE '12), pp. 622626, Chengdu, China, June 2012.

[23] G.-H. Zhou, C.-C. Zuo, J.-Z. Wang, and S.-X. Liu, "Gearbox fault diagnosis based on wavelet-AR model," in Proceedings of the 6th International Conference on Machine Learning and Cybernetics (ICMLC '07), vol. 2, pp. 1061-1065, Hong Kong, August 2007.

[24] N. K. Nikhar, S. S. Patankar, and J. V. Kulkarni, "Gear tooth fault detection by autoregressive modelling," in Proceedings of the International Conference on Computing, Communications and Networking Technologies (ICCCNT '13), pp. 1-6, Tiruchengode, India, July 2013.

[25] F. J. Villalobos-Piña and R. Alvarez-Salas, "Robust algorithm for electric fault diagnosis in the three phase induction machine based on spectral and wavelet tools," Revista Iberoamericana de Automatica e Informatica Industrial, vol. 12, no. 3, pp. 292-303, 2015.

[26] D. L. Milanez and A. E. Emanuel, "The instantaneous-spacephasor: a powerful diagnosis tool," IEEE Transactions on Instrumentation and Measurement, vol. 52, no. 1, pp. 143-148, 2003.

[27] X. Lin, Y. Xie, D. Zhao, and S. Xu, "Estimation of observer parameters for dynamic positioning ships," Mathematical Problems in Engineering, vol. 2013, Article ID 173603, 7 pages, 2013.

[28] J. G. Proakis and D. G. Manolakis, Digital Signal Processing, Pearson Prentice Hall, New Jersey, NJ, USA, 2007.

[29] M. H. Hayes, Statistical Digital Signal Processing and Modeling, John Wiley \& Sons, New York, NY, USA, 1996.
[30] A. Neumaier and T. Schneider, "Estimation of parameters and eigenmodes of multivariate autoregressive models," ACM Transactions on Mathematical Software, vol. 27, no. 1, pp. 27-57, 2001.

[31] H.-C. Wu, S. Y. Chang, T. Le-Ngoc, and Y. Wu, "Efficient rankadaptive least-square estimation and multiple-parameter linear regression using novel dyadically recursive hermitian matrix inversion," International Journal of Antennas and Propagation, vol. 2012, Article ID 891932, 10 pages, 2012.

[32] M. Drif and A. J. M. Cardoso, "Airgap-eccentricity fault diagnosis, in three-phase induction motors, by the complex apparent power signature analysis," IEEE Transactions on Industrial Electronics, vol. 55, no. 3, pp. 1404-1410, 2008. 


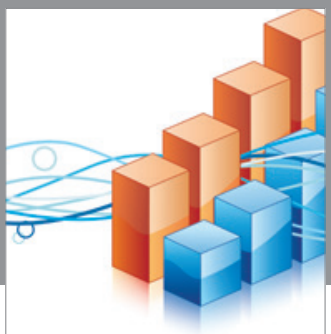

Advances in

Operations Research

vatem alat4

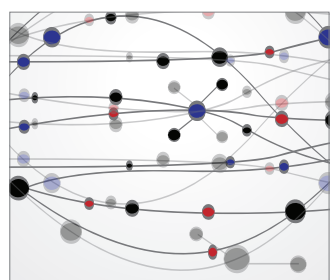

\section{The Scientific} World Journal
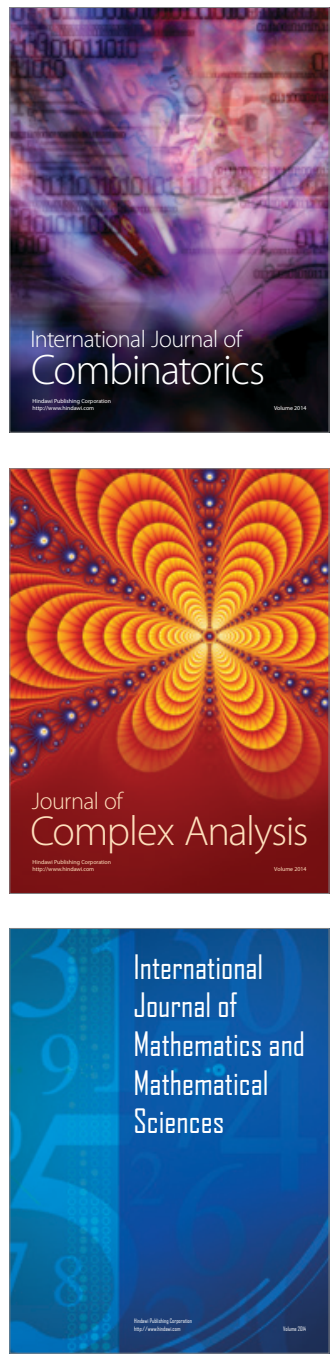
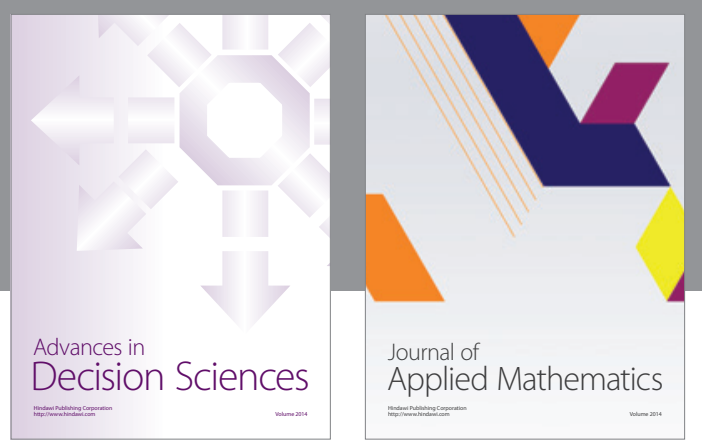

Algebra

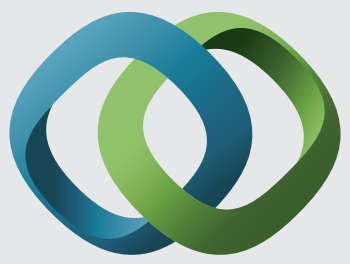

\section{Hindawi}

Submit your manuscripts at

http://www.hindawi.com
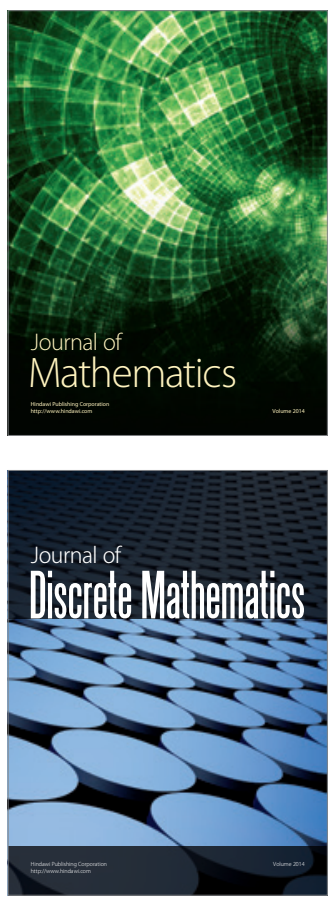

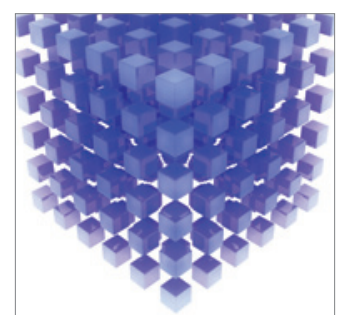

Mathematical Problems in Engineering
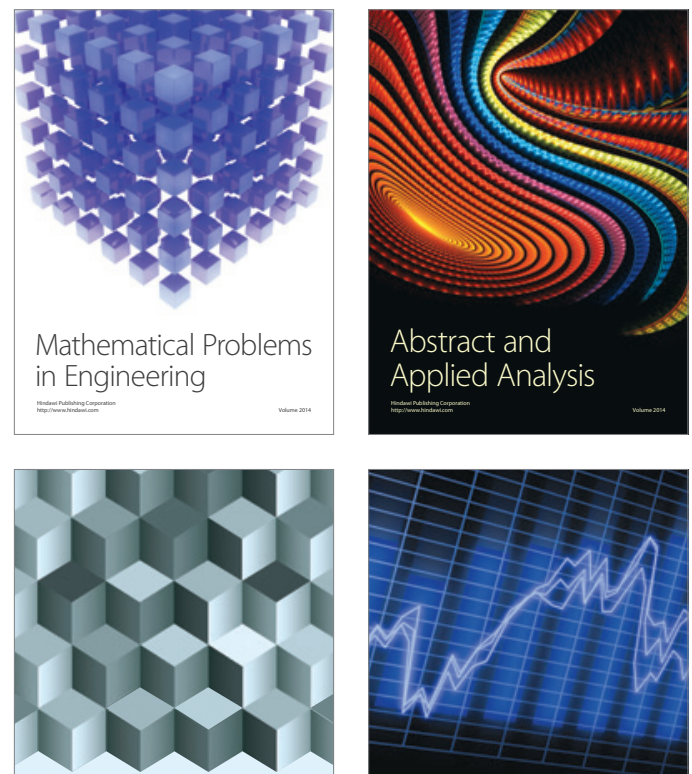

Journal of

Function Spaces

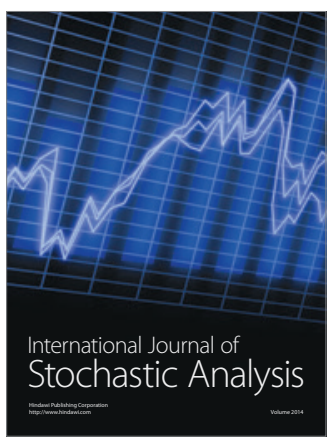

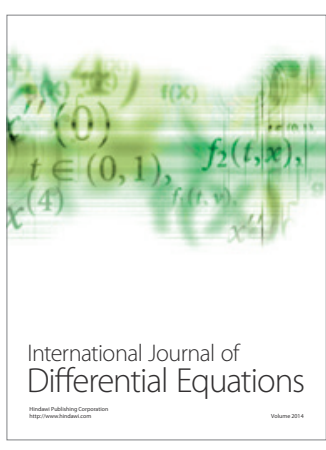
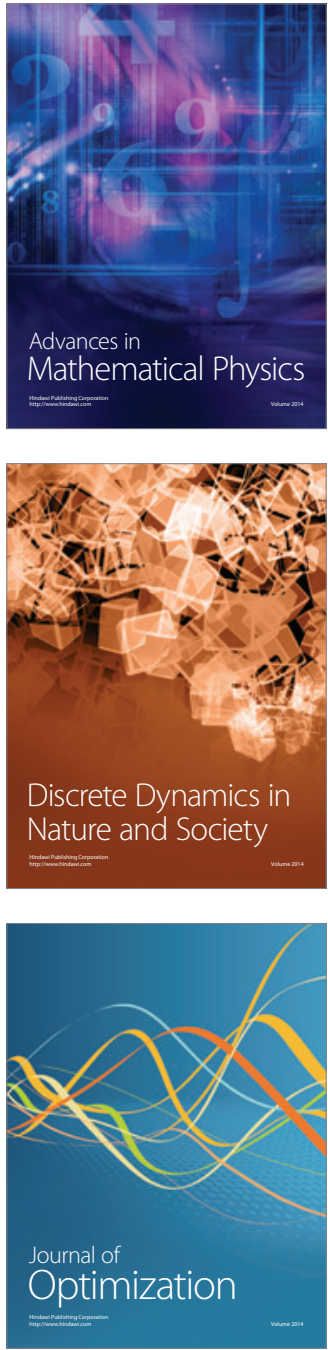